\title{
Help to Intercept Hepatoma Risk--May your Liver be Smooth Please
}

\author{
Raghavendra Rao MV1*, Kumar P1, Sateesh Babu A1, Sripada \\ Pallavi T22, Krishna Sowmya $\mathbf{M}^{3}$, Ramanaiah CJ4, Mahindra KV5, \\ Siddharth Sri Gokul $\mathbf{K}^{6}$, Amin $\mathrm{F}^{1}$ and Sireesha Bala $\mathbf{A}^{1}$ \\ ${ }^{1}$ Avalon University School of Medicine, Curacao, Central America \\ ${ }^{2}$ APollo Institute of Medical Science and Research Institute, Jubilee Hills, Hyderabad, \\ Telangana, India
}

${ }^{3}$ Burjeel Hospital, Abu Dhabi, United Arab Emirates

${ }^{4}$ Amina Hospital Sharjah, United Arab Emirates

${ }^{5}$ Acharya Nagarjuna University, Guntur, Andhra Pradesh, India

${ }^{6}$ Pondicherry Institute of Medical Sciences, Pondicherry, India

*Corresponding author: MV Raghavendra Rao, Professor \& Head of the Department of Diseases, Immunity and Therapeutics (DIT), Dean (Student affairs) \& Research Director, Avalon University School of Medicine, Sta. Rosaweg 122124, Willemstad, Curacao, Central America, Tel: +5999 788-8008; Email: dr.raghavendra@avalonu.org

\begin{abstract}
"MAY YOUR LIVER BE SMOOTH". Liver is the "hot organ" and is mainly concerned with digestion. Liver cancer is most common in countries located in sub-Saharan Africa and Southeast Asia. Cancer is the leading cause of death in Canada. It is estimated that one in two Canadians will be diagnosed with cancer during their lifetime. Cancer is a fatal disease which may occur in a person at any point in his life due to the abnormal growth of cells. Liver cancer is one such type of cancer that occurs due to the occurrence of tumors in the liver. We are developing new cutting-edge therapies using cellular and genetic engineering to fight cancer. These therapies not only represent the next generation of cancer treatments, but the underlying technologies will help create new medicines for use beyond cancer and significantly impact other areas from clean energy to food production. Using microwave ovens, drinking from plastic bottles and consuming food additive are causes of cancer, believing a "worrying" number of peoples. The findings come from a survey in which 1,330 people in England were quizzed on risk factors. The results show that $40 \%$ wrongly thought stress and food additives cause cancer.
\end{abstract}

Keywords: Hepatocellular carcinoma (HCC); Inducible nitric oxide synthase (iNOS); Inflammatory cytokines; Tumor necrosis factor (TNF)- $\alpha$; Interleukin (IL)-1 $\beta$; Gadolinium-enhanced MRI; Non-alcoholic fatty liver disease (NAFLD); Alpha fetoprotein (AFP) 


\section{Medical Journal of Clinical Trials \& Case Studies}

\section{Introduction}

Hepatocellular carcinoma (HCC) is the most common primary malignancy of the liver. The global burden of cancer in 2012 was an all-time high of 14 million cases and is predicted to grow to 22 million over the next two decades [1]. Hepatocellular carcinoma (HCC) is one of the leading causes of cancer related to worldwide death with a great geographical variation [2]. To be eligible for curative therapy at the time of diagnosis is important. However, the majority of cases are diagnosed at late stages. This can be achieved with applicable screening modalities. Liver cancer is the fifth most common cancer in men and the seventh in women [3]. Worldwide incidence is between 250,000 and 1,000,000 new cases per year with a male-female ratio of about 4 or $5: 1$ [4]. Hepatocellular carcinoma (HCC) is the most often primary cancer of the liver, accounting from $85 \%$ till $90 \%$ of all primary liver cancers [5].

The principal treatment is surgical resection or liver transplantation, depending on whether the patient is a suitable transplant candidate HCC development, in most cases, arises in the setting of underlying end-stage liver disease, secondary to either viral hepatitis (specifically hepatitis B virus (HBV) or hepatitis C virus) or other nonviral chronic liver diseases [6,7]. Over time, the underlying etiologies, incidence, and HCC outcomes are changed according to the countries $[8,9]$. While the incidence of HCC is rising in the west, attributed to the past HCV epidemic (baby-boomers) and trends of metabolic disorders, it is decreasing in the East $[10,11]$.

In the inflamed liver, there is an increased production of nitric oxide (NO) by inducible nitric oxide synthase (iNOS), and inflammatory cytokines such as tumor necrosis factor (TNF)- $\alpha$ and interleukin (IL)-1 $\beta$, which have been implicated as factors in liver injury Chronic hepatitis and cirrhosis of any cause accounts for 70-90\% cases of HCC occurring on a background of chronic liver disease [12,13]. Human immunodeficiency virus coinfection with either HBV or hepatitis C virus (HCV) leads to quicker progression to cirrhosis and HCC Guidelines for HCC were recently developed according to the GRADE approach [6,14,15]. The Guidelines for HCC were developed using clinically relevant questions, which were then answered by systematic reviews of the literature, and followed by data-supported recommendations. Cirrhosis was estimated to cause over 1.2 million deaths ( $2 \%$ of global deaths) in 2013 , an increase of $47 \%$ since $1990[16,17]$.
Cirrhosis and HCC are the major life-limiting consequences of progressive fibrotic liver diseases, mainly caused by hepatitis B virus (HBV), hepatitis $C$ virus (HCV), alcohol abuse, and non-alcoholic fatty liver disease (NAFLD). HCC mortality rates have been increasing across almost all counties over the past three decades, particularly in white men aged 55 to 64 years old with HCV infection, and Hispanics affected by NAFLD in the Texas region $[18,19]$.

\section{History}

The number of new cases of hepatocellular carcinoma per year in India in males is about 4.1 and for females 1.2 per 100,000. It typically occurs between 40 and 70 years of age. Liver cancer is the eighteenth most common cancer in the UK, and it is the twelfth most common cause of cancer death [20]. Liver cancer death rates for adults aged 25 and over increased 43 percent from 7.2 per 100,000 U.S [21]. The death rate for men was between 2.0-2.5 times the rate for women throughout this period. Globally, as of 2010, liver cancer resulted in 754,000 deaths, up from 460,000 in 1990, making it the third leading cause of cancer death after lung and stomach [22]. In 2012 , it represented $7 \%$ of cancer diagnoses in men, the $5^{\text {th }}$ most diagnosed cancer that year China has $50 \%$ of HCC cases globally, and more than $80 \%$ of total cases occur in sub-Saharan Africa or in East-Asia due to hepatitis B virus Cholangiocarcinoma also has a significant geographical distribution, with Thailand showing the highest rates worldwide due to the presence of liver fluke [6,23-25].

\section{Significant Gapin Research}

Since the 1970s, the epidemic of hepatocellular carcinoma (HCC) has spread beyond the Eastern Asian predominance and has been increasing in the Northern hemisphere, especially in the United States (US) and Western Europe. It occurs more commonly in males in the fourth and fifth decades of life. The incidence of the nonalcoholic fatty liver disease has been on the rise and it has also been associated with the development of HCC Hepatocellular carcinoma (HCC) is the most often primary cancer of the liver and is one if the leading cause of cancer-related death worldwide [26]. Patients with hepatocellular carcinoma have poor prognosis despite the achievements in surgery techniques and other therapeutic procedures and it is a reason why continuous attention should be paid to this issue [27]. 


\section{Medical Journal of Clinical Trials \& Case Studies}

Hepatocellular carcinoma (HCC) is among the leading causes of cancer-related mortality. The principal treatment is surgical resection or liver transplantation, depending on whether the patient is a suitable transplant candidate. However, in most patients with HCC the diagnosis is often late, thereby excluding the patients from definitive surgical resection. Due to the poor prognosis of patients with HCC, newer treatments are needed with several being in development, either in preclinical or clinical studies. L-Carnitine has protective effects on various injured organs. However, it has not been reported whether L-carnitine influences the induction of inducible nitric oxide synthase (iNOS) expression during inflammation [7]. Nitric oxide (NO) produced by iNOS is an inflammatory indicator in organs which become inflamed, including the liver. The treatment of hepatocellular carcinoma (HCC) remains a challenge, with 1- and 3-year survival rates of $20 \%$ and $5 \%$, respectively, and a median survival of 8 months [28]. However, a better understanding of the pathogenesis of HCC, and advances in targeted molecular therapies provide physicians treating this disease with new hope. A dietary carcinogen, aflatoxin, may be a cofactor for hepatocellular carcinoma, especially in Africa and China [29]. The advent of an effective hepatitis $B$ vaccine for the prevention of primary infection raises the possibility of prevention of hepatocellular carcinoma, particularly in areas of the world where infection with hepatitis B virus is hyper endemic (e.g. Africa, China, and Southeast Asia). When it infects B lymphocytes of susceptible humans. Normal human lymphocytes have a limited lifespan in vitro, but EB virus can immortalize such lymphocytes into lymphoblast cell lines that grow indefinitely in culture.

\section{Major Advances and Discoveries}

Hepatocellular carcinoma (HCC) is the fifth most common malignancy, the third most common cause of cancer death, and the most common primary liver cancer. Therefore, metabolite analysis in blood or urine should lead to the detection of suitable candidates for the detection of HCC [30]. This guidance provides a datasupported approach to the diagnosis, staging, and treatment of patients diagnosed with hepatocellular carcinoma (HCC). Patients who develop the chronic fibrotic liver disease, caused by viral or metabolic etiologies, are at a high risk of developing hepatocellular carcinoma (HCC) [31]. Even after complete HCC tumor resection or ablation, the carcinogenic tissue microenvironment in the remnant liver can give rise to recurrent de novo HCC tumors, which progress into incurable, advanced-stage disease in most patients. Thus, early detection and prevention of HCC development is, in principle, the most impactful strategy to improve patient prognosis.

However, a "one-size-fits-all" approach to HCC screening for early tumor detection, as recommended by clinical practice guidelines, is utilized in less than $20 \%$ of the target population, and the performance of screening modalities, including ultrasound and alpha-fetoprotein, is suboptimal. Furthermore, optimal screening strategies for emerging at-risk patient populations, such as those with chronic hepatitis $\mathrm{C}$ after a viral cure, or those with noncirrhotic, non-alcoholic fatty liver disease remain controversial. Hepatocellular carcinoma (HCC) occurs in up to $5 \%$ of cirrhotic patients per year [32]. There has been a rise in the incidence of HCC in the United States over the past few decades, which is likely secondary to an increased prevalence of NAFLD, HCV infections, and chronic hepatitis B infections due to Immigration from countries with a high prevalence of HBV.

Several etiologic factors have been identified in the development of tumor. Malignant transformation is heightened in any form of chronic liver disease, particularly cirrhosis. The risk of developing HCC is increased 100-fold in chronic HBV carriers, even in the absence of cirrhosis. In the setting of chronic HBV infection, $30-50 \%$ of cases of HCC occur in the absence of cirrhosis. The risk of developing HCC in the setting of HCV cirrhosis approximately $1-4 \%$ per year. Mycotoxinsmetabolites of saprophytic fungi-are known hepatic carcinogens and have been proposed to act synergistically with cirrhosis and HBV infection in increasing the risk of liver cell cancer. Hormonal factors have been implicated by experimental studies. The tumor is known to have a male predominance. In addition to causing hepatitis, hepatitis B virus is a risk factor in the development of liver cancer in humans [33].

Epidemiologic and laboratory studies have proved persistent infection with hepatitis $B$ virus to be an important cause of chronic liver disease and the development of hepatocellular carcinoma. Hepatitis B virus infections occurring in adults are usually resolved, but primary infections in neonates and young children tend to become chronic in up to $90 \%$ of cases. It is these persistent hepatitis B virus infections in neonates and young children tend to become chronic in up to $90 \%$ of cases. It is these persistent hepatitis $B$ virus infections established early in life that carry the 


\section{Medical Journal of Clinical Trials \& Case Studies}

highest risk of hepatocellular carcinoma later in life. Woodchucks are an excellent model for hepatitis B virus infections in humans. A similar virus, woodchuck hepatitis virus, establishes chronic infections in both newborn and adult woodchucks, many of which develop hepatocellular carcinomas within a 3-year period. Hepatitis C virus, a member of the Flaviviridae family, contains a genome of single-stranded RNA $9.4 \mathrm{~kb}$ in size. It appears that the majority of the infections become persistent, even in adults. Chronic infection with hepatitis $\mathrm{C}$ virus is also considered to be a causative factor in hepatocellular carcinoma. Most probably, hepatitis $\mathrm{C}$ virus acts indirectly in the development of hepatocellular carcinoma.

It is clear that viruses involved in the genesis of several types of human tumors. Proving a causal relationship between a virus and a given type of cancer is, in general, difficult. Only if the continued expression of a viral infection is necessary for the maintenance of transformation will viral genes necessarily persist in every tumor cell. Conversely, a virus may be found associated frequently with a tumor but be there simply as a passenger because of an affinity for the cell type [34].

\section{Where the Research Go Next?}

One of the long goals for cancer research has been the discovery of a universal blood test that can detect the presence of the disease long before any of the symptoms appear. Such a test have elusive, but it took a step closer towards becoming a reality with the news back in January that trials at John Hopkins University School of Medicine in Baltimore of a blood test they call Cancer SEEK produced some dramatic results.

The idea behind a blood test for the disease is that it looks for traces of faulty DNA that cancer cells release into the bloodstream. The advantages of this are that it would be quicker and less invasive than other tests, such as biopsies, and rather less expensive. Detecting the disease earlier also opens the way for quicker treatment, which is more likely to be successful treatment. If you take these proteins by themselves they may not tell you much, but put them together and the signal becomes much stronger. Nobody has managed to look for so many cancer types before. But because you are focusing on eight types rather than one specific cancer, this makes it more challenging. It's easier to make mistake". With the test formulated to search for the ctDNA and protein markers, the team applied it to 1,005 people already diagnosed with cancer that had progressed to either stage two or three. Across all the types of cancer, the test detected 70 out of 100 cases [35].

\section{Current Debate}

The success of the screening is influenced by the availability of effective treatment with the identification of the target population and the selection of appropriate screening tests. The cost-effectiveness should also be taken into consideration. Nearly $85 \%-95 \%$ of HCC is developed on the cirrhotic liver [36,37]. These patients have a lifetime risk of developing HCC by $30 \%$ with leading cause of liver-related death in compensated cirrhosis. Following the introduction of sorafenib for the treatment of HCC, Phase III trials of numerous other agent as first-line or second-line chemotherapy have been conducted to determine if any of these agents might offer superior survival benefit to sorafenib $[38,39]$. Unlike the other hepatocellular adenomas, which are comprised of only hepatocytes and vessels with minor amounts of stroma, these molecules may also be elevated in the serum [40]. $10 \%$ of these HCAs that also have $\beta$-catenin activating mutations and, as would be anticipated, also show increased nuclear levels of $\beta$-catenin by immunohistochemistry. Much less common are carcinomas of bile duct origin, Cholangiocarcinoma [41].

\section{Tumor Markers}

AFP is a serum tumor marker for HCC; however, it is only increased in approximately one-half of U.S. patients. The lens culinary agglutinin-reactive fraction of AFP (AFP-L3) assay is thought to be more specific. The other widely used assay is that for des- $\gamma$-carboxy prothrombin (DCP), a protein induced by vitamin $\mathrm{K}$ absence (PIVKA-2). This protein is increased in as many as $80 \%$ of HCC patients but may also be elevated in patients with vitamin K deficiency; it is always elevated after Coumadin use. $\mathrm{T}$ may predict for portal vein invasion. Both AFP-L3 and DCP are FDA-approved. Standard liver function tests should be performed including prothrombin time (PT), partial thromboplastin time (PTT), albumin, transaminases, $\gamma$ glutamyl trans peptidase, and alkaline phosphatase. Decreases in platelet count and white blood cell count may reflect portal hypertension and associated hypersplenism.

Hepatitis A, B, and C serology should be measured. Portal vein invasion is normally detected as an obstruction and expansion of the vessel. A chest CT is used to exclude metastases. MRI can also provide detailed 


\section{Medical Journal of Clinical Trials \& Case Studies}

information, especially with the newer contrast agents. Ethiodol (Lipiodol) is an methodized oil emulsion retained by liver tumors that can be delivered by hepatic artery injection (5-15 mL) for CT imaging 1 week later. For small tumors, Ethiodol injection is very helpful before biopsy because the histological presence of the dye constitutes proof that the needle biopsied the mass under suspicion. A prospective comparison of triphasic CT, gadolinium-enhanced MRI, ultrasound, and fluorodeoxyglucose positron emission tomography (FDGPET) showed similar results for CT, MRI, and ultrasound; PET imaging was unsuccessful [42].

In 1963, Abelev and colleagues in Moscow reported that in chemically-induced and transplantable hepatomas, mice and rats synthesized and secreted AFP into blood. In the following year, Tatarinov reported raised serum AFP in six patients with hepatocellular cancer (HCC). In 1967, Abelev and associates in a landmark article described more fully the clinical importance of AFP in HCC in a larger study using agar precipitation and immunoelectroporosis (IEP), and reported that serum AFP was also raised in non-seminomatous germ cell (NSGC) tumors of testis or ovary but not in some other testicular tumors [43].

\section{Conclusion}

Hepatocellular carcinoma is an aggressive tumor associated with a poor prognosis. Given its asymptomatic nature in the early stages, HCC is mostly diagnosed at advanced stages, often leading to incurable clinical situations. Current and prior treatment options were only modestly associated with increased survival. In fact, the survival benefit with sorafenib was only for a few months. Nowadays, newly emerging therapeutic targets, as well as new drugs and therapeutic modalities have been investigated in pre-clinical and clinical trials using microwave ovens, drinking from plastic bottles and consuming food additive are causes of cancer, believing a "worrying" number of peoples [7]. Researchers from University College London and the University of Leeds carried out the survey. Samuel Smith, from the University of Leeds, said" "It's worrying to see so many people's endorse risk factors for which there is no convincing evidence"."Compared to past research, it appears the number of people believing in unproven causes of cancer has increased since the start of the century" [44].

\section{References}

1. Stuver S, Trichopoulos D (2008) Cancer of the liver and biliary tract. In: Adami HO, Hunter D, Trichopoulos D (Eds.), Textbook of Cancer Epidemiology. 2nd (Edn.), New York: Oxford University Press.

2. Stewart BW, Wild CP (2014) editors. World Cancer Report 2014. Lyon, France: International Agency for Research on Cancer.

3. Nevin Yilmaz, Ugur Eser Yilmaz, Kaya Suer, Vedat Goral, Nedim Cakir (2018) Screening for hepatocellular carcinoma: summary of current guidelines up to 2018. Hepatoma Res 4: 46.

4. El-Serag HB (2011) Hepatocellular Carcinoma. N Engl J Med 365: 1118-1127.

5. Simonetti RG, Liberati A, Angiolini C, Pagliaro L (1997) Treatment of hepatocellular carcinoma: A systematic review of randomized controlled trials. Ann Oncol 8(2): 117-136.

6. El-Serag HB, Rudolph KL (2007) Hepatocellular Carcinoma: Epidemiology and Molecular Carcinogenesis. Gastroenterology 132(7): 2557-2576.

7. Daher S, Massarwa M, Benson AA, Khoury T (2018) Current and Future Treatment of Hepatocellular Carcinoma: An Updated Comprehensive Review. J Clin Transl Hepatol 6(1): 69-78.

8. Singal AG, El-Serag HB (2015) Hepatocellular carcinoma from epidemiology to prevention: translating knowledge into practice. Clin Gastroenterol Hepatol 13(12): 2140-2151.

9. Gomes MA, Priolli DG, Tralhão JG, Botelho MF (2013) Hepatocellular carcinoma: epidemiology, biology, diagnosis, and therapies. Rev Assoc Med Bras (1992) 59(5): 514-524.

10. Altekruse SF, Henley SJ, Cucinelli JE, Mc Glynn KA (2014) Changing hepatocellular carcinoma incidence and liver cancer mortality rates in the United States. Am J Gastroenterol 109(4): 542-553.

11. Global Burden of Disease Liver Cancer Collaboration, Akinyemiju T, Abera S, Ahmed M, Alam N, et al. (2017) The burden of primary liver cancer and underlying etiologies from 1990 to 2015 at the global, regional, and national level: Results From the Global 


\section{Medical Journal of Clinical Trials \& Case Studies}

Burden of Disease Study 2015. JAMA Oncol 3(12): 1683-1691.

12. Colasanti M, Suzuki H (2000) The dual personality of NO. Trends Pharmacol Sci 21(7): 249-252.

13. Iwakiri Y, Kim MY (2015) Nitric oxide in liver diseases. Trends Pharmacol Sci 36(8): 524- 536.

14. Macdonald DC, Nelson M, Bower M, Powles T (2008) Hepatocellular carcinoma, human immunodeficiency virus and viral hepatitis in the HAART era. World J Gastroenterol 14(11): 1657-1663.

15. This recommendation is intended for patients with decompensated cirrhosis who are already on the LT waitlist-and thus with an indication for transplantation in addition to HCC-and is based on current organ allocation policies in the United States. Future allocation policy revisions may impact this recommendation.

16. The choice of observation with follow-up imaging versus treatment depends on several factors, including patient preference, anticipated waiting time, rate of growth of the lesion, degree of liver decompensation, and AFP.

17. GBD Mortality and Causes of Death C (2015) Global, regional, and national age-sex specific all-cause and cause-specific mortality for 240 causes of death, 1990-2013: a systematic analysis for the Global Burden of Disease Study 2013. Lancet 385(9963): 117-171.

18. Higashi T, Friedman SL, Hoshida Y (2017) Hepatic stellate cells as key target in liver fibrosis. Adv Drug Delivery Rev 121: 27-42.

19. Mokdad AH, Dwyer-Lindgren L, Fitzmaurice C, Stubbs RW, Bertozzi Villa A, et al. (2017) Trends and patterns of disparities in cancer mortality among us counties, 1980-2014. JAMA 317(4): 388-406.

20. Overview of Hepatocellular Carcinoma (2017) Liver Cancer.

21. Liver cancer statistics (2014) Cancer Research UK.

22. Xu J (2018) Trends in Liver Cancer Mortality Among Adults Aged 25 and Over in the United States, 20002016. NCHS Data Brief (314): 1-8.
23. Lozano R, Naghavi M, Foreman K, Lim S, Shibuya K, et al. (2012) Global and regional mortality from 235 causes of death for 20 age groups in 1990 and 2010: a systematic analysis for the Global Burden of Disease Study 2010. Lancet 380(9859): 2095-2128.

24. World Cancer Report (2014) International Agency for Research on Cancer. World Health Organization.

25. Khan SA, Toledano MB, Taylor-Robinson SD (2008) Epidemiology, risk factors, and pathogenesis of cholangiocarcinoma. HPB 10 (2): 77-82.

26. Ghouri YA, Mian I, Rowe JH (2017) Review of hepatocellular carcinoma: Epidemiology, etiology, and carcinogenesis. J Carcinog 16: 1.

27. Dafina Janevska, Viktorija Chaloska-Ivanova, Vlado Janevski (2015) Hepatocellular Carcinoma: Risk Factors, Diagnosis and Treatment. Open Access Maced J Med Sci 3(4): 732-736.

28. Yusuke Nakamura, Hiroya Iida, Richi Nakatake, Tatsuma Sakaguchi, Masaki Kaibori, et al. (2018) LCarnitine has a liver-protective effect through inhibition of inducible nitric oxide synthase induction in primary cultured rat hepatocytes. Functional Foods in Health and Disease 8(3): 212-227.

29. Sonja K Olsen, Robert S Brown Jr, Abby B Siegel (2010) Hepatocellular carcinoma: review of current treatment with a focus on targeted molecular therapies. Therap Adv Gastroenterol 3(1): 55-66.

30. Caroline R Cartlidge, M R Abellona U, Alzhraa M A Alkhatib, Simon D Taylor-Robinson (2017) The utility of biomarkers in hepatocellular carcinoma: review of urine-based $1 \mathrm{H}-\mathrm{NMR}$ studies - what the clinician needs to know. International Journal of General Medicine 10: 431-442.

31. Marrero JA, Kulik LM, Sirlin CB, Zhu AX, Finn RS, et al. (2018) Diagnosis, Staging, and Management of Hepatocellular Carcinoma: 2018 Practice Guidance by the American Association for the Study of Liver Diseases. Hepatology 68(2): 723-750.

32. Fujiwara N, Friedman SL, Goossens N, Hoshida Y (2018) Risk factors and prevention of hepatocellular carcinoma in the era of precision medicine. J Hepatology 68(3): 526-549. 


\section{Medical Journal of Clinical Trials \& Case Studies}

33. Stephen J McPhee, Gary D Hammer. Pathophysiology of Disease. $6^{\text {th }}$ (Edn.), Mc Graw Hill Lange, pp: 408409.

34. Geo F Brooks, Janet S Butel, Stephen A Morse. Jawetz, Melnick and Adelberg's Medical Microbiology. 23 ${ }^{\text {rd }}$ (Edn.), Mc Graw Hill Lange, pp: 602-603.

35. The Medical Scientist, IBMS, Institute of Biomedical Science, Cristian Tomasetti, University of Maryland (US), pp: 16-17.

36. Llovet JM, Burroughs A, Bruix A (2003) Hepatocellular carcinoma. Lancet 362(9399): 19071917.

37. Forner A, Reig M, Bruix J (2018) Hepatocellular carcinoma. Lancet 391(10127): 1301-1314.

38. Sangiovanni A, Prati GM, Fasani P, Ronchi G, Romeo R, et al. (2006) The natural history of compensated cirrhosis due to hepatitis $C$ virus: a 17-year cohort study of 214 patients. Hepatology 43(6): 1303-1310.
39. Hung TH, Liang CM, Hsu CN, Tai WC, Tsai KL, et al. (2017) Association between complicated liver cirrhosis and the risk of hepatocellular carcinoma in Taiwan. PLoS One 12: e0181858.

40. Masafumi Ikeda, Chigusa Morizane, Makoto Ueno, Takuji Okusaka, Hiroshi Ishii, et al. (2018) Chemotherapy for hepatocellular carcinoma: current status and future perspectives Japanese Journal of Clinical Oncology 48(2,1): 103-114.

41. Kumar, Abbas and Aster. Robbins and Cotran Pathologic basis of Disease. 9th (Edn.), Elsevier Saunders, pp: 869.

42. Longo, Fauci, Kasper, Hauser and Jameson. Harrison's Principles of Medicine, $18^{\text {th }}$ (Edn.), Chapter 1-223, 1: 779-780.

43. The Biomedical Scientist (2018) Institute of Biomedical Science, pp: 30.

44. The Biomedical Scientist (2018) Institute of Biomedical Science, pp: 9.

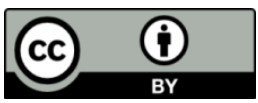

